\title{
Reporte de las serpientes del municipio de Tamalameque, Cesar - Colombia
}

\section{Snake report of the municipality of Tamalameque, Cesar - Colombia}

\author{
Oscar Ruiz P, * Biólogo.
}

Gobernación del Cesar. Secretaria de Educación Departamental. Institución Educativa Ernestina Pantoja. Área de Ciencias Naturales. Tamalameque, Colombia. *Correspondencia: oscarruiz1980@ hotmail.com.

Recibido: Junio de 2010; Aceptado: Agosto de 2011.

\section{RESUMEN}

Objetivo. Identificar preliminarmente los ofidios del municipio de Tamalameque, departamento del Cesar-Colombia. Materiales y métodos. Entre enero y mayo de 2009, mediante búsqueda libre y captura manual se efectuaron muestreos de serpientes, con dos muestreos por mes y un esfuerzo de captura de cuatro horas/hombres. Resultados. Se reporta para esta localidad la presencia de tres familias de serpientes, distribuidas en 12 géneros y 13 especies; La familia Colubridae fue la mejor representada con el $76.93 \%$ de las especies reportadas, seguida de la familia Boidae $15.38 \%$ y Anomalepidae $7.69 \%$. Conclusiones. Los resultados permiten deducir que la familia Colúbridae es un componente herpetológico importante para la Ciénaga del Cristo y que las amenazas antropicas para los ofidios en esta localidad son la destrucción de hábitat y falta de conocimiento ecológico y etológico por parte de los pobladores

Palabras clave: Serpientes, identificación, muestreo aleatorio (Fuente:AIMS).

\begin{abstract}
Objective. Preliminary identification of the ophidians of the municipality of Tamalameque, department of Cesar Colombia. Materials and methods. Between January and May of 2009, by means of free search and manual capture snake samplings were taken, with two samplings per month and a capture effort of four man-hour. Results. The presence of three snake families is reported for this locality, distributed in 12 genera and 13 species. The Colubridae family was the best represented one with the $76.93 \%$ of the reported species, followed by $15.38 \%$ Boidae and $7.69 \%$ Anomalepidae families. Conclusions. The results allow to deduce that the family Colubridae is an important herpetological component of the "Cienaga del Cristo" and that anthropic threats for ophidians in this locality are habitat destruction and lack of ecological and ethological knowledge by the community.
\end{abstract}

Key Words: snakes, identification, random sampling (Source:AIMS). 


\section{INTRODUCCIÓN}

Se puede afirmar que las serpientes son lagartos especializados (1). Las serpientes carecen de oído y no pueden captar sonidos, los ojos carecen de párpados y no tienen extremidades, el maxilar y la mandíbula están articulados por ligamentos, lo que les permite tragar presas muy grandes (2). Para la región Caribe Sánchez et al (3), registraron seis familias distribuidas en 31 géneros y 48 especies. En total para Colombia se estiman 589 especies de reptiles, de los cuales aproximadamente 250 de estas son serpientes (4).

El objetivo del presente estudio fue identificar preliminarmente los ofidios del municipio de Tamalameque, departamento del CesarColombia. Con este trabajo se contribuye al conocimiento de la ofidio fauna de la región Caribe colombiana ya que se reporta la presencia de 13 especies para el municipio de Tamalameque asociadas a las inmediaciones de la Ciénaga del Cristo en el departamento del Cesar.

\section{MATERIALES Y MÉTODOS}

Sitio de estudio. El municipio de Tamalameque se encuentra dentro de las coordenadas geográficas extremas $8^{\circ} 11^{\prime}$ de latitud Norte, 72030' de longitud oeste del meridiano de Greenwich, cuya extensión es de $400 \mathrm{Km}^{2}$, presentándose una altura sobre el nivel del mar de 100 a 150 m. Al sur el municipio es bañado por la aguas del río Magdalena, el cual es responsable de la formación de varias ciénagas presentes en la zona, una de las cuales es la ciénaga del Cristo. La mayor parte de los muestreos realizados se llevaron a cabo en inmediaciones de este cuerpo de agua. Este ecosistema se caracteriza por la oferta de nichos que posee debido a los cambios que presenta a lo largo del ciclo anual de aguas altas y bajas.

Muestreos. Los muestreos se realizaron dos veces al mes, en los meses de eneromayo de 2009, mediante la metodología de búsqueda libre y captura manual. Durante los recorridos se revisaron diferentes microhabitats (riachuelos, hojarasca, debajo de rocas, árboles caídos, borde de ciénaga, huecos en el suelo y en árboles, etc); en los muestreos también se tuvieron en cuenta especímenes hallados muertos o eliminados por los pobladores. En total se llevaron a acabo 10 muestreos, cada salida con un esfuerzo de captura de 4 horas/hombre, algunos ejemplares fueron preservados según lo estipulado por Páez (4). El material colectado fue determinado usando las claves taxonómicas de Janis A Roze (5) y Carlos Pérez Santos (6).

Trabajo con la comunidad. A la par de los muestreos también se hicieron talleres de sensibilización con jóvenes de la comunidad, especialmente estudiantes de la Institución educativa Ernestina Pantoja, ubicada en la cabecera municipal, los jóvenes después de varias charlas donde se les mostraron especímenes vivos y conservados, para examinarlos y manipularlos; tuvieron la oportunidad de establecer diferencias morfológicas entre especies venenosas y no venosas. También se les dio información sobre el papel ecológico que desempeñan los ofidios en los lugares que habitan.

\section{RESULTADOS}

Se reporta para el municipio de Tamalameque la presencia de tres familias de ofidios, distribuidas en 12 géneros y 13 especies (Tabla 1). Algunas especies registradas son: Boa Constrictor, Thamnodynastes gambotensis, Liophis lineatus, entre otras (Figura 1). La familia Colubridae representó el $76.93 \%$ de las especies reportadas, boidae el $15.38 \%$ y Anomalepidae el $7.69 \%$ (Figura 2).

Tabla 1. Serpientes registradas para el Municipio de Tamalameque, Departamento del CesarColombia.

\begin{tabular}{llll}
\hline Familia & Genero & Especie & $\begin{array}{l}\text { Nombre } \\
\text { común }\end{array}$ \\
\hline Colubridae & $\begin{array}{l}\text { Leptophis } \\
\text { Leptodeira }\end{array}$ & $\begin{array}{l}\text { L. ahaetulla } \\
\text { L. septentrionalis }\end{array}$ & $\begin{array}{l}\text { Bejuquilla } \\
\text { Mapana }\end{array}$ \\
& Liophis & L. melanotus & Guarda camino \\
& Oxybelis & O. lineatus & Guarda camino \\
& Spilotes & S. pullatus & Bejuquillo \\
& Pseudoboa & S. neuwiedii & Coral \\
& Thamnodynastes & T. gambotensis & Patoco \\
& Helicops & H. danieli & Mapana de agua \\
& Chironius & C. carinatus & Amarilla \\
Boa & B. constrictor & Boa \\
& Epicrates & E. cenchria & Candelilla \\
Liotyphlops & L. albirostris & ciega \\
\hline
\end{tabular}




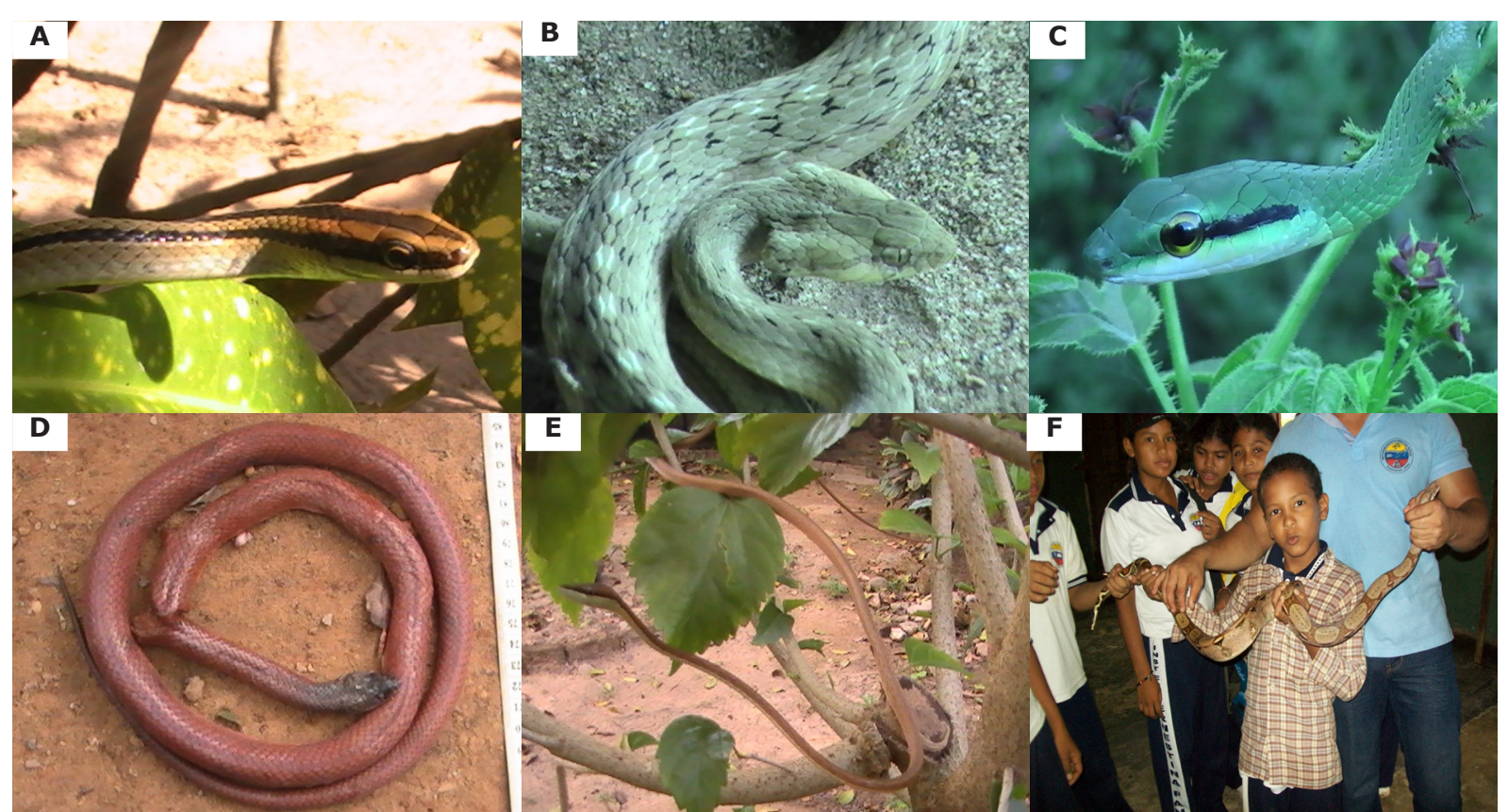

Figura 2. Fotografías de algunos de los ofidios registrados en Tamalameque-Colombia. A) Liophis linneatus; B) Thamnodynastes gambotensis; C) Leptophis ahaetulla; D) Pseudoboa neuwiedii; E) Oxybelis aeneus; F) Boa constrictor.

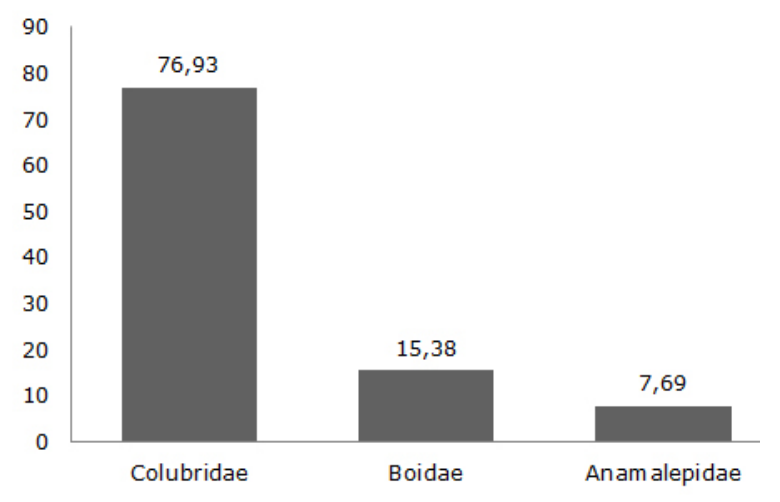

Figura 1. Porcentaje de especies por familia de serpientes reportados para Tamalameque, Cesar-Colombia.

\section{DISCUSIÓN}

El hecho de que la familia colubridae tuviera la mayor representación de especies, se podría explicar por las siguientes condiciones: la distribución de esta familia a nivel mundial es alta e incluye el $70 \%$ de todas las especies de serpientes del mundo (7) y además, esta familia se caracteriza por tener un amplio rango de habitats y hábitos alimentarios (6); son predadoras que pueden cazar ranas, sapos, lagartijas, peces, renacuajos, pájaros, mamíferos, moluscos, insectos e incluso otras serpientes (8).
En la zona se presenta el caso de tráfico ilegal de fauna con la especie Boa constrictor (Boidae), la cual es cazada por los pobladores para ser vendida a intermediarios, el destino de estos animales es ser usados en la industria del cuero o ser vendidos como mascotas. Según información suministrada por cazadores una hembra preñada puede ser vendida entre U\$25 y U\$30. En Colombia el trafico de reptiles para el 2002 representó el 59\% del tráfico ilegal de fauna, mientras que aves y mamíferos sumados, representaron $21 \%$ (9).

El panorama para la otra especie de boa (Epicrates cenchria) reportada en la región no es alentador, debido a su coloración café-rojizo, los pobladores la llaman candelilla, atribuyéndole la capacidad de envenenar y matar; esta creencia errónea hace que esta especie sea exterminada. En general la amenaza más grave que enfrentan las serpientes en esta localidad es la pérdida de hábitat. En cuanto al número de especies reportadas, es posible que si se realizan trabajos donde se hagan muestreos durante todo el ciclo anual de aguas altas y bajas de la Ciénaga del Cristo, el número de estas para la localidad aumente, debido a que los cambios en la fisionomía del paisaje pueden ofrecer diversidad de nichos.

La percepción que tienen los pobladores acerca de las serpientes, es parecida a la que se presenta en el resto de la región Caribe, miedo y desconocimiento, ya que para la mayor 
parte de los miembros de la comunidad todas son venenosas y deben ser eliminadas de los ecosistemas; por lo tanto se enseñó a las comunidad que los ofidios son componentes importantes en los ecosistemas, ya que actúan como controladores de poblaciones de otras especies, y además, son fuente de alimento para otros organismos. Según Seigel (10) en muchas partes del mundo las serpientes son importantes debido a que poseen un amplio rango de especializaciones.

Los talleres tuvieron gran acogida y despertó en los jóvenes el interés de saber más acerca de las serpientes y su importancia para los ecosistemas; con las actividades desarrolladas se logró enseñar a los estudiantes las características morfológicas que diferencian a las serpientes venenosas y no venenosas. El contacto directo con algunas especies colectadas permitió un acercamiento, que en muchos casos derrumbó falsas creencias negativas que giraban en torno a las serpientes y cambió la forma de pensar de muchos jóvenes. Alrededor de 300 estudiantes, de los grados 6 - 11 participaron en los talleres, los cuales se desarrollaron como parte de la asignatura de ciencias naturales y medio ambiente.

En conclusión, los resultados permiten deducir que la familia Colúbridae es un componente herpetológico importante para la Ciénaga del Cristo y que las amenazas antropicas para los ofidios en esta localidad son la destrucción de hábitat y falta de conocimiento ecológico y etológico por parte de los pobladores

\section{Agradecimientos}

A los estudiantes de los diferentes grados que participaron activamente en los muestreos, al señor David Noriega, coordinador de la institución académica Ernestina Pantoja, por su apoyo incondicional y a Maideth Romero por su ayuda continúa.

\section{REFERENCIAS}

1. Bauer AM. Lizard. Reptiles and Amphibians. New York, USA: Editores Cogger $\mathrm{H}$ and Zweifel R; 1992.

2. Renjifo JM Y Lundberg M. Anfibios y reptiles de Urrá. Medellín, Colombia: Editorial Colonia; 1999.

3. Sánchez H, Castaño-Mora O, \& CárdenasArévalo G. Colombia diversidad biótica I. Bogotá, Colombia: Editorial Guadalupe Ltda; 1995.

4. Páez VP, Bock BC, Estrada JJ, Ortega Á, Daza Juan M, Gutiérrez PD. Guía de campo de algunas especies de anfibios y reptiles de Antioquia. Medellín, Colombia: Multiimpresos Ltda; 2002.

5. Roze JA. La taxonomía y zoogeografia de los ofidios de venezuela. Caracas, Venezuela: Ediciones de la Biblioteca UCV; 1996.
6. Pérez-Santos C. Las serpientes del Atlántico. Madrid-España: Museo Nacional de Ciencias Naturales; 1986.

7. Poug HF, Andrews RM, Cadle JE, Crump MI, Savitzky AH, Wells kD. Herpetology. New Jersey, USA: Prentice-Hall Inc; 1998.

8. Otero-Patiño R. Manual de diagnóstico y tratamiento ofídico. Medellín, Colombia: Editorial Universidad de Antioquia; 1994.

9. Acopazoa y fondo para la acción ambiental. Biodiversidad. Colombia país de vida. Cali, Colombia: Cargraphics S.A: 2004.

10. Seigel RA, Collins JT, Novak S. Snakes: Ecology and Evolutionary Biology. New York NY: MacMillan Publishing Co; 1987. 